\title{
Desempenho tardio das biopróteses valvulares porcinas
}

\author{
Marcos Vinícius H. de CARVALHO*, Reinaldo W. VIEIRA*, Januário M. SOUZA**, Sérgio Almeida de

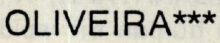

\section{RBCCV 44205-159}

CARVALHO, M. V. H.; VIEIRA, R. W.; SOUZA, J. M.; OLIVEIRA, S. A. - Desempenho tardio das biopróteses valvulares porcinas. Rev. Bras. Cir. Cardiovasc., 7(1):28-37,1992.

RESUMO: O objetivo deste trabalho foi o de estudar o desempenho tardio das biopróteses porcinas, modelo Carpentier-Edwards, com ênfase a todos os eventos mórbidos e/ou letais que pudessem estar relacionados à presença da bioprótese. Foram estudados 100 pacientes consecutivos submetidos a substituição de valva mitral e 100 pacientes consecutivos submetidos a substituição de valva aórtica. 0 seguimento médio dos pacientes foi de 93 meses para pacientes submetidos a substituiçäo da valva mitral e de 62 meses para os pacientes submetidos a substituição de valva aórtica. Aproximadamente, $80 \%$ dos pacientes permaneceram vivos ao longo do seguimento. As curvas de sobrevida dos pacientes submetidos a substituição de valva mitral e dos pacientes submetidos a substituiçäo de valva aórtica demonstram um descenso inicial em razäo da mortalidade hospitalar e, a partir daí, as curvas se estabilizam, voltando a ter outro descenso a partir dos cinco a seis anos da cirurgia, provavelmente em razão da alta incidência de degeneraçāo estrutural nesse período. Os pacientes que necessitaram reoperação para substituir a bioprótese que sofreu deterioração estrutural tiveram mortalidade maior do que aqueles que não necessitaram reoperação. Entretanto, essa diferença não teve significância estatística. A mortalidade relacionada à presença da bioprótese foi de aproximadamente $5 \%$, tanto nos portadores de bioprótese em posição mitral, quanto nos portadores de bioprótese em posição aórtica. Entre os pacientes que receberam implante da bioprótese em posição mitral, 22 necessitaram reoperação para substituir a bioprótese, sendo que a incidência de reoperação foi maior nos pacientes que tinham menos de 35 anos de idade na ocasiāo da primeira operação. Vinte e dois pacientes submetidos a substituição de valva mitral necessitaram reoperação, enquanto que apenas sete pacientes submetidos a substituição de valva aórtica necessitaram reoperação. As complicações trombo-embólicas foram raras com o uso das biopróteses, apesar dos pacientes não terem recebido anticoagulação oral sistêmica. Concluímos que: 1) o uso das biopróteses porcinas tipo CarpentierEdwards, em nosso meio, apresentou resultados clínicos satisfatórios, com mortalidade hospitalar e tardia semelhante à de outros grupos e também semelhante àquela quando outros substitutos valvulares são empregados; 2) a deterioração estrutural é um evento marcante para os pacientes portadores dessas biopróteses, e começa a ocorrer basicamente a partir de seis a sete anos após o implante; 3 ) a deterioração estrutural da bioprótese modifica o destino dos pacientes, motivando a reoperação para substituir a bioprótese implantada. Entretanto, a ocorrência desta reoperação não aumenta, significativamente, a mortalidade.

DESCRITORES: próteses valvulares cardíacas, biológicas.

\section{INTRODUÇÃO}

A cirurgia de substituição valvar tem salvado muitas vidas e proporcionado, a um grande número de pacientes, uma vida mais saudável e produtiva. Esses fatos levam à conclusão de que os pacientes com doença cardíaca valvar, em sua maioria, possam ser considerados candidatos potenciais à cirurgia ${ }^{22}$.

A valvula biológica mais amplamente utilizada tem sido a valvula aórtica porcina montada em anel

Trabalho realizado no Serviço do Prof. Dr. Sérgio Almeida de Oliveira. Hospital da Beneficiência Portuguesa. São Paulo, SP, Brasil.

Recebido para publicação em 15 de fevereiro de 1992.

* Do Hospital do Servidor Público Estadual de São Paulo.

** Do Hospital das Clínicas da Universidade de Campinas.

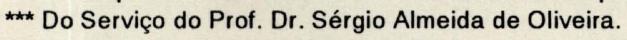

Endereço para separatas: Marcos Vinícios de Carvalho. Caixa Postal 483. 13.270 Valinhos, SP, Brasil. 
CARVALHO, M. V. H.; VIEIRA, R. W.; SOUZA, J. M.; OLIVEIRA, S. A. - Desempenho tardio das biopróteses valvulares porcinas. Rev. Bras. Cir. Cardiovasc., $7(1): 28-37,1992$.

flexível. A mesma é confeccionada utilizando-se válvula aórtica de porco preservada em glutaraldeído, sendo a bioprótese modelo Carpentier-Edwards uma das mais utilizadas. Essa bioprótese foi desenhada para implantação em posição mais supra-anular do que propriamente no anel valvare, assim, permitir um diâmetro do orifício da prótese de mesmas dimensões que o diâmetro do anel valvar do paciente. $\mathrm{Na}$ sua confecção, é usado um suporte flexível, no qual o tecido da válvula aórtica porcina é suturado e fixado com glutaraldeído a baixa pressão ${ }^{5}$.

Desde que a cirurgia de substituição valvar se tornou um procedimento rotineiro e com baixa mortalidade hospitalar, fez-se necessário conhecer qual o desempenho das biopróteses ao longo do tempo. $\mathrm{Na}$ evolução dos pacientes dever-se-ia conhecer a sobrevivência, o desempenho hemodinâmico, a durabilidade das biopróteses e os fatores negativos relacionados à presença de bioprótese (trombo-embolismo, endocardite infecciosa, degeneração estrutural, degeneração não-estrutural, mortalidade e morbidade e combinação de conseqüências mórbidas). Várias publicaçōes foram surgindo, discorrendo sobre a evolução dos pacientes submetidos a substituição valvar a médio e a longo prazo. Entretanto, ficou evidente que a mortalidade e a morbidade dos pacientes que se submeteram ao implante da bioprótese dependiam também de fatores outros relacionados ao paciente; por exemplo, disfunção ventricular esquerda, coronariopatia associada, paciente idoso e doença multivalvar ${ }^{20}$. Se as complicaçōes próteserelacionadas eram mais em função do substrato de pacientes submetidos ao implante do que da prótese em si, tornava-se necessária a elaboração de um protocolo. Permitir-se-ia que as diferentes séries de pacientes pudessem ser comparadas com o propósito de se facilitar a análise dos resultados das operações de substituição valvar e propiciando a comparação entre as experiências de diferentes cirurgiöes, os quais operam diferentes séries de pacientes com técnicas e biopróteses diferentes. Assim, a American Association for Thoracic Surgery publicou o "Guidelines for Reporting Morbility and Mortality after Cardiac Valvular Operations" 11.

A substituição de uma valva cardíaca por uma prótese metálica ou bioprótese oferece bons resultados hemodinâmicos e pode ser realizada com baixo risco imediato. Entretanto, na evolução tardia, os pacientes podem apresentar novos problemas próteserelacionados, tendo-se em vista que o substituto valvar ideal ainda não foi obtido. O grande número de próteses valvulares existentes demonstra as suas imperfeiçōes, a necessidade de pesquisas adicionais e o desenvolvimento de novos modelos ${ }^{10}$.

As biopróteses porcinas têm sido o substituto valvar preferido, em nosso Serviço, nos últimos anos, em razão das dificuldades encontradas em nosso meio com o uso sistemático e adequado de anticoagulante.

O presente trabalho tem como objetivo estudar o desempenho tardio das biopróteses valvulares porcinas modelo Carpentier-Edwards, em posição mitral e em posição aórtica, e a evolução dos pacientes, com ênfase a todos os eventos mórbidos e/ou letais que pudessemestar relacionados à presença da bioprótese.

\section{CASUÍSTICA E MÉTODOS}

Para este estudo foram considerados apenas os primeiros 100 pacientes submetidos a substituição valvar mitral e os primeiros 100 pacientes submetidos a substituição valvar aórtica, com o intuito de se conseguir um maior período de seguimento de pacientes.

Com a intenção de se diminuir a interferência de fatores relacionados diretamente ao paciente, foram excluídos aqueles operados dentro do período do estudo, mas que necessitaram procedimentos associados, como: substituição valvar dupla ou tripla e revascularização miocárdica.

Para análise da evolução hospitalar, foram revisados os prontuários dos pacientes. $A$ análise da evolução tardia baseou-se em entrevista direta com o paciente, quando possível. Outra forma de análise foi por contato pelo correio, através de questionário dirigido enviado ao médico cardiologista que acompanhava o paciente. Quando nenhuma forma de avaliação foi possível, fez-se contato pelo telefone com o paciente, ou com sua família. No caso de óbito, as informações relacionadas com a causa da morte foram fornecidas pelo cardiologista que estava dando seguimento clínico, pelos familiares e/ou através do atestado de óbito.

\section{Substituição da Valva Mitral}

Foram considerados os primeiros 100 pacientes consecutivos, operados entre maio de 1979 e junho de 1981, para troca de valva mitral por bioprótese porcina, os quais foram acompanhados de maneira já abordada. O seguimento pós-operatório foi de 79 a 103 meses (média de 93 meses). Desses 100 pacientes, 38 eram do sexo masculino e 62 do sexo feminino (razão homens/mulheres de 0,6:1). Houve perda de evolução de dois pacientes. A idade variou entre $6 \mathrm{e}$ 70 anos, sendo que 31 pacientes tinham menos de 35 anos na ocasião da cirurgia.

Considerando-se o diagnóstico anatômico da lesão, 46 pacientes tinham estenose da valva mitral, 32 tinham dupla disfunção mitral e 22 tinham insuficiência valvar. 
CARVALHO, M. V. H.; VIEIRA, R. W.; SOUZA, J. M.; OLIVEIRA, S. A. - Desempenho tardio das biopróteses valvulares porcinas. Rev. Bras. Cir. Cardiovasc., 7(1):28-37,1992.

As lesōes foram provocadas por doença reumática em 94 pacientes, degeneração mixomatosa em 5 e por isquemia em 1 paciente. Näo houve caso em que a cirurgia por troca de valva mitral fosse decorrente de endocardite infecciosa, nesse grupo. Entre esses 100 pacientes, nenhum foi operado em classe funcional I (NYHA), 5 estavam em classe funcional II, 59 em classe funcional III e 36 em classe funcional IV.

\section{Substituição da Valva Aórtica}

Foram considerados os primeiros 100 pacientes consecutivos operados para troca da valva aórtica, no período compreendido entre novembro de 1979 e novembro de 1983. O seguimento pós-operatório foi de 44 a 92 meses (média de 62 meses). Oitenta e três pacientes eram do sexo masculino e 17 do sexo feminino (coeficiente homens/mulheres de 4,81:1). A idade variou entre 14 e 74 anos. Desses 100 pacientes, 31 deles também tinham menos de 35 anos de idade na data de operação. Houve perda da evolução de 3 pacientes. As lesões valvares foram estenose aórtica em 32 pacientes, insuficiência aórtica em 38 e dupla disfunção aórtica em 30 pacientes. A etiologia dessas lesões foi doença reumática em 49 pacientes, calcificação aórtica idiopática em 39, degeneração mixomatosa em 11 e lesão aórtica pós-endocardite infecciosa em 1 paciente. A distribuição por classe funcional (NYHA), na ocasião da cirurgia, foi de 4 pacientes em classe funcional II, 53 em classe funcional III e 43 em classe funcional IV.

\section{Técnica Cirúrgica}

Todos os pacientes foram operados através de toracotomia mediana anterior com esternotomia longitudinal. A canulação arterial foi feita através da aorta ascendente, a venosa através da cânula única atrial para substituição da valva aórtica e canulação de ambas as veias cavas para a substituição da valva mitral. Empregou-se, para circulação extracorpórea, oxigenador de bolhas, hemodiluição total com perfusato de 1,5 litro de solução de Ringer lactato e hipotermia sistêmica a $28^{\circ} \mathrm{C}$. A proteção miocárdica consistiu na infusão de solução cardioplégica cristalóide, hipercalêmica e hipotérmica $\left(4^{\circ} \mathrm{C}\right)$ com soluçāo de St. Thomas ${ }^{4}$. O saco pericárdico foi mantido preenchido com soro gelado durante o período de parada cardíaca anóxica. Na substituição da valva aórtica, o átrio esquerdo foi drenado através da veia pulmonar superior direita e foi feita aortotomia oblíqua em direçāo ao seio de Valsalva não coronariano. A valva aórtica foi removida e a bioprótese foi implantada com fixação através de pontos separados em $U$, passados do ventrículo para a aorta e sempre ancorados em pequenas almofadas de feltro de Teflon. A sutura da aortotomia foi em chuleio simples contí- nuo com fio de Polipropileno quatro zeros. A substituição da valva mitral foi realizada através de atriotomia esquerda, anteriormente às veias pulmonares direitas. Nos 20 primeiros pacientes submetidos a substituição da valva mitral, foi realizada proteção miocárdica com solução cardioplégica de St. Thomas injetada na aorta ascendente através de cateter $14 \mathrm{~F}$. A partir do vigésimo primeiro paciente desta série, todos os demais foram operados com parada cardíaca anóxica por pinçamentos aórticos intermitentes de até dez minutos, intercalados com períodos de aproximadamente três minutos de perfusão coronária.

A valva mitral foi totalmente removida nos casos de estenose pura ou predominante; nos casos de insuficiência valvar pura ou predominante, a cúspide posterior foi mantida junto às cordas tendíneas e seus músculos papilares. As biopróteses foram fixadas no anel mitral com pontos separados de Poliester multifilamentado dois zeros, ancorados em almofadas de feltro de Teflon, passados do átrio para o ventrículo. A atriotomia foi suturada com fios de Propileno três zeros em chuleio simples.

\section{Definição das Complicações Relacionadas à Cirurgia e à Prótese}

As informaçōes obtidas sobre os pacientes foram organizadas e enquadradas conforme o proposto pelo "Guidelines for Morbility and Mortality after Cardiac Valvular Operations" ${ }^{11}$.

\section{Metodologia Estatística}

Como primeira fase, apresentamos uma análise descritiva de variáveis, como tempo de sobrevivência, idade e censura (falha, sobrevivência e perdidos). Ainda nessa fase, utilizamos o teste $T$ de Student, para estudar a relação entre o grupo e a idade, para verificar se os grupos são homogêneos quanto à idade e utilizar teste do "log-rank" para verificar o efeito da idade na sobrevivência.

$\mathrm{Na}$ segunda etapa, estudamos as funcões de sobrevivência dos pacientes com substituição das valvas mitral e aórtica, reoperados ou não, através da tabela de curvas de sobrevivência (produto-limite de Kaplan-Meier). Realizamos, ainda, teste de "log-rank", para verificar as diferenças entre os tempos de sobrevivência desses grupos.

\section{RESULTADOS}

A análise das curvas de sobrevivência mostra que aproximadamente $80 \%$ dos pacientes estão vivos ao longo do seguimento de 5 a 6 anos após o implante da bioprótese, tanto em posicão mitral, 
CARVALHO, M. V. H.; VIEIRA, R. W.; SOUZA, J. M.; OLIVEIRA, S. A. - Desempenho tardio das biopróteses valvulares porcinas.

Rev. Bras. Cir. Cardiovasc., 7(1):28-37,1992.

quanto em posicão aórtica. Também observamos que há um descenso inicial da curva em razão da mortalidade hospitalar e, a partir daí, a curva se estabiliza, voltando a apresentar outro descenso a partir de cinco a seis anos após a cirurgia original. A análise das curvas também demonstra que a sobrevivência foi maior entre aqueles pacientes que não necessitaram reoperação do que entre aqueles que necessitaram ser reoperados por deterioração da bioprótese implantada. Entretanto, essa diferença não tem significância estatística (Figuras 1 e 2).

As Figuras 3 e 4 referem-se às curvas de sobrevivência dos pacientes que se submeteram a implante de bioprótese em posição mitral e que tinham idade abaixo e acima de 42,5 anos. Esta idade, que dividiu

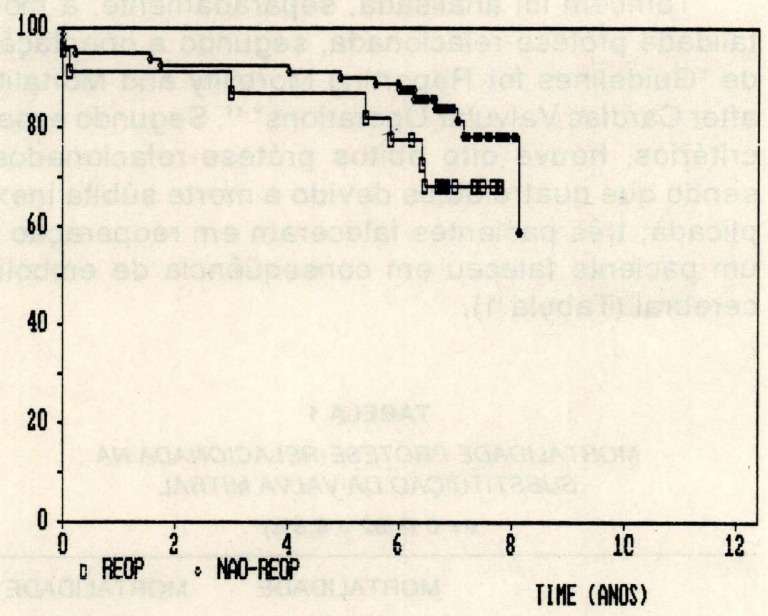

Fig. 1 - Curvas de sobrevivência dos pacientes submetidos a substituição de valva mitral, comparando os que necessitaram ou não reoperação durante o seguimento.

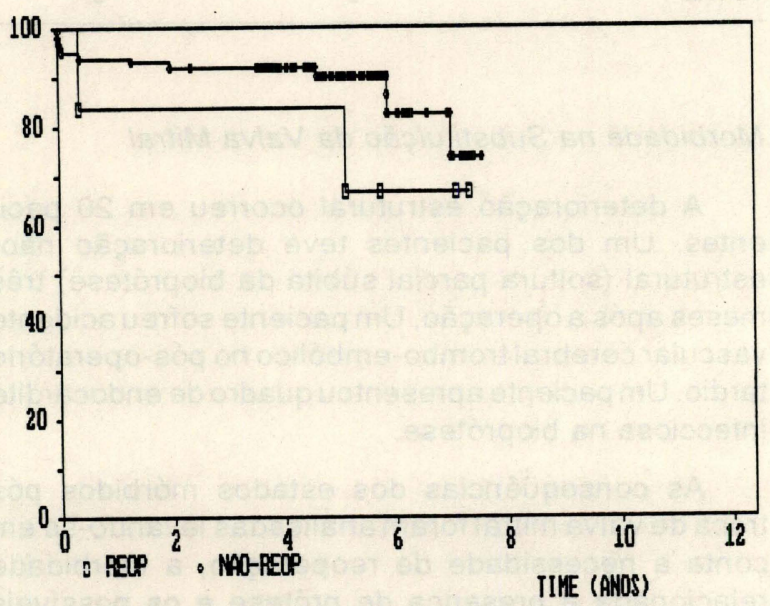

Fig. 2 - Curvas de sobrevivência dos pacientes submetidos a substituição de valva aórtica, comparando os que necessitaram ou não reoperação durante o seguimento.

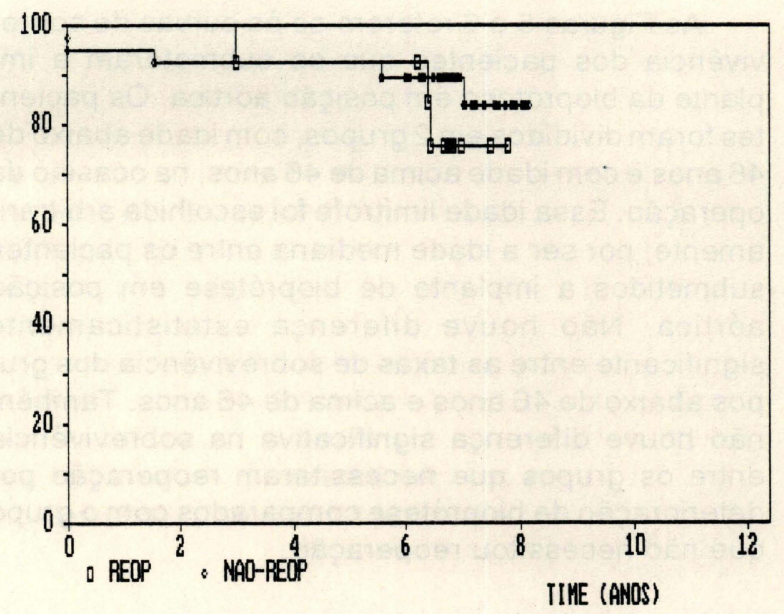

Fig. 3-Curvas de sobrevivência dos pacientes submetidos a substituição de valva mitral e que tinham idade inferior a 42,5 anos na ocasião da cirurgia, comparando os que necessitaram ou não reoperação durante o seguimento.

os pacientes em 2 grupos (abaixo de 42,5 anos e acima de 42,5 anos), foi arbitrariamente escolhida por ser a idade mediana dos pacientes que se submeteram a implante da bioprótese em posição mitral. Embasados na análise dessas curvas de sobrevivência, podemos observar que a mortalidade é um pouco menor nos pacientes que não necessitaram reoperação para substituição de bioprótese, quando comparados com os pacientes que necessitaram da reoperação. $A$ análise estatística referente à taxa de sobrevivência não mostra diferença de significância estatística entre esses grupos.

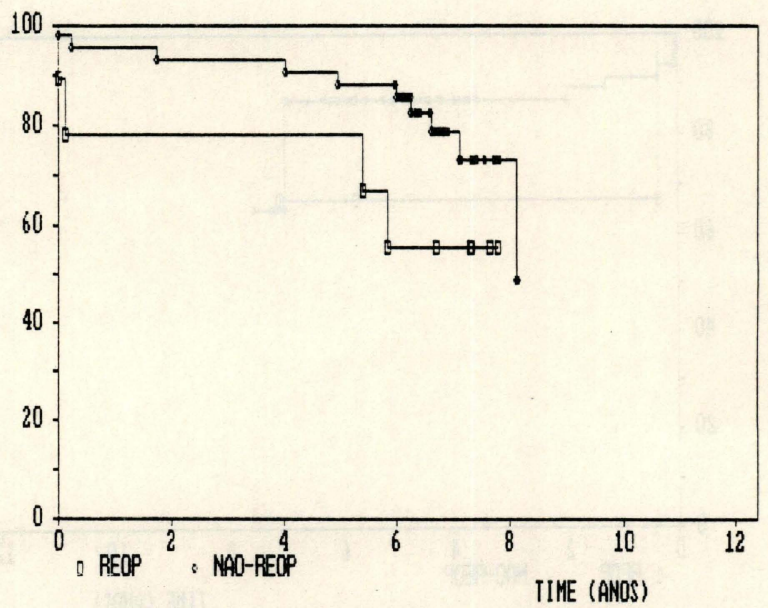

Fig. 4 - Curvas de sobrevivência dos pacientes submetidos a substituição de valva mitral e que tinham idade superior a 42,5 anos na ocasião da cirurgia, comparando os que necessitaram ou não reoperação durante o período de seguimento. 
CARVALHO, M. V. H.; VIEIRA, R. W.; SOUZA, J. M.; OLIVEIRA, S. A. - Desempenho tardio das biopróteses valvulares porcinas. Rev. Bras. Cir. Cardiovasc., 7(1):28-37,1992.

As Figuras 5 e 6 referem-se às curvas de sobrevivência dos pacientes que se submeteram a implante da bioprótese em posição aórtica. Os pacientes foram divididos em 2 grupos, com idade abaixo de 46 anos e com idade acima de 46 anos, na ocasião da operação. Essa idade limítrofe foi escolhida arbitrariamente, por ser a idade mediana entre os pacientes submetidos a implante de bioprótese em posição aórtica. Não houve diferença estatisticamente significante entre as taxas de sobrevivência dos grupos abaixo de 46 anos e acima de 46 anos. Também não houve diferença significativa na sobrevivência entre os grupos que necessitaram reoperação por deterioraçāo da bioprótese comparados com o grupo que não necessitou reoperação.

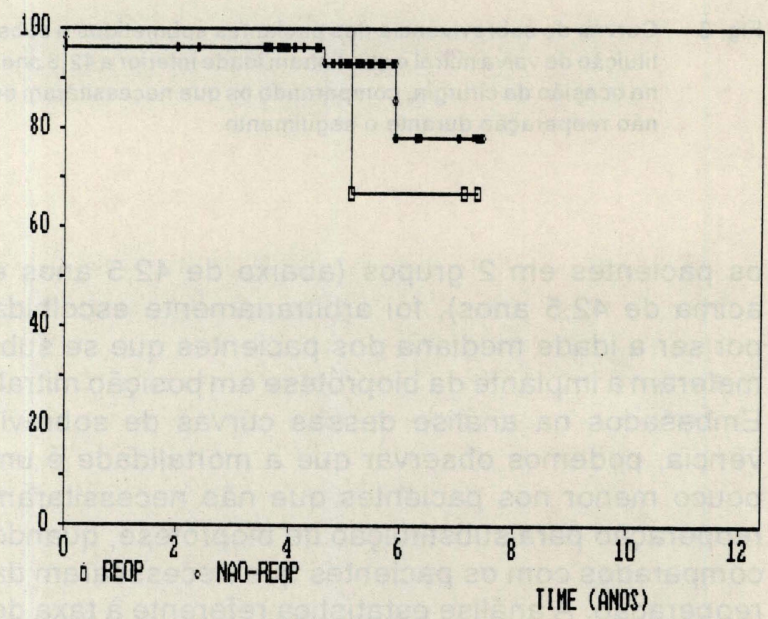

Fig. 5 - Curvas de sobrevivência dos pacientes submetidos a substituição de valva aórtica e que tinham idade inferior a 46 anos na ocasião da cirurgia, comparando os que necessitaram ou nảo reoperação durante o período de seguimento.

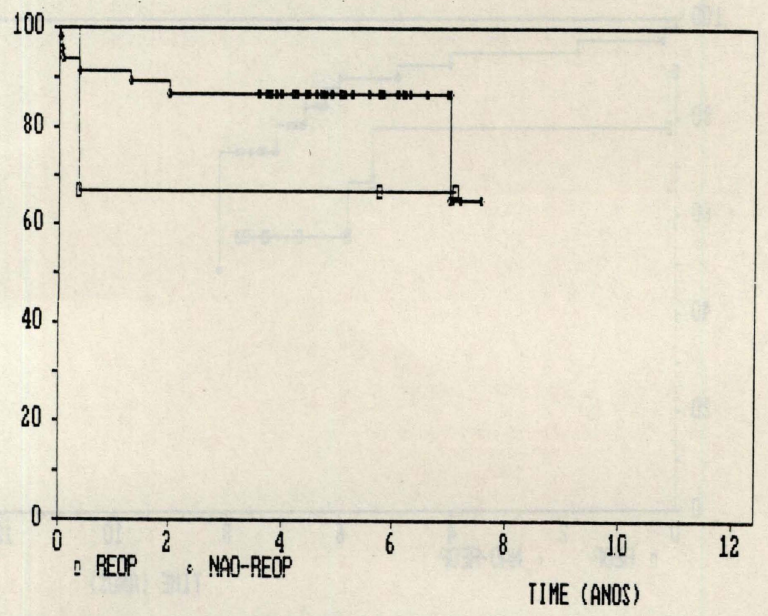

Fig. 6-Curvas de sobrevivência dos pacientes submetidos a substituição de valva aórtica e que tinham idade superior a 46 anos na ocasião da cirurgia, comparando os que necessitaram ou não reoperação durante o período de seguimento.

\section{Mortalidade na Substituição da Valva Mitral}

Os óbitos imediatos ocorreram em seis pacientes, sendo que em quatro a morte foi em decorrência de falência miocárdica e em dois por parada cardíaca súbita não recuperada. Os óbitos imediatos ocorreram nos grupos funcionais III e IV. Não houve diferenças apreciáveis entre o tipo de lesão anátomo-funcional tratada e mortalidade hospitalar, sendo 3 óbitos entre os 46 pacientes portadores de estenose valvar, 2 óbitos entre os 32 pacientes com dupla disfunção e 1 óbito entre os 22 pacientes com insuficiência valvar.

A mortalidade tardia, durante o período de seguimento, foi de $17,3 \%$ (16/92) (houve perda do seguimento de 2 pacientes e 6 óbitos imediatos).

Também foi analisada, separadamente, a mortalidade prótese-relacionada, segundo a orientação de "Guidelines for Reporting Morbility and Mortality after Cardiac Valvular Operations" "11. Segundo esses critérios, houve oito óbitos prótese-relacionados, sendo que quatro deles devido a morte súbita inexplicada; três pacientes faleceram em reoperação e um paciente faleceu em conseqüência de embolia cerebral (Tabela 1).

TABELA 1

MORTALIDADE PRÓTESE-RELACIONADA NA SUBSTITUIÇÃO DA VALVA MITRAL

$\mathrm{n}=8(8 / 92=8,6 \%)$

\begin{tabular}{lcc}
\hline & $\begin{array}{c}\text { MORTALIDADE } \\
\text { IMEDIATA }\end{array}$ & $\begin{array}{c}\text { MORTALIDADE } \\
\text { TARDIA }\end{array}$ \\
\hline Morte Súbita Inexplicada & 2 & 2 \\
Óbito em Reoperações & - & 3 \\
Embolia Cerebral & - & 1 \\
\hline TOTAL & 2 & 6 \\
\hline
\end{tabular}

\section{Morbidade na Substituição da Valva Mitral}

A deterioração estrutural ocorreu em 20 pacientes. Um dos pacientes teve deterioração nãoestrutural (soltura parcial súbita da bioprótese) três meses após a operação. Um paciente sofreu acidente vascular cerebral trombo-embólico no pós-operatório tardio. Um paciente apresentou quadro de endocardite infecciosa na bioprótese.

As conseqüências dos estados mórbidos pós troca de valva mitral foram analisadas levando-se em conta a necessidade de reoperação, a morbidade relacionada à presença de prótese e os possíveis prejuízos permanentes relacionados à prótese. Dos 92 pacientes acompanhados, 22 deles necessitaram reoperação, sendo que um paciente necessitou ser 
CARVALHO, M. V. H.; VIEIRA, R. W.; SOUZA, J. M.; OLIVEIRA, S. A. - Desempenho tardio das biopróteses valvulares porcinas. Rev. Bras. Cir. Cardiovasc., 7(1):28-37,1992.

reoperado por 2 vezes. Alguns autores sugerem que, sendo as biopróteses mais propensas a sofrerem deterioração nos pacientes mais jovens, elas não devem ser usadas em pacientes com idade inferior a $30-35$ anos ${ }^{14-18}$. Assim, procuramos dividir arbitrariamente os pacientes submetidos a substituição de valva mitral em 2 grupos com idade inferior e superior a 35 anos. A proporção de pacientes que necessitaram substituir a bioprótese devido a deterioração e que tinham idade menor ou igual a 35 anos $(37,0 \%)$ foi maior que a proporção de pacientes que necessitaram substituir a bioprótese e que tinham idade superior a 35 anos $(18,4 \%)$. Entretanto, o valor crítico encontrado no teste do Qui quadrado foi de 0,057 , o que está muito próximo de $5 \%$ (Tabela 2 ).

\section{TABELA 2}

VALVA MITRAL: DISTRIBUICÃO DOS PACIENTES QUE NECESSITARAM OU NÃO REOPERAÇÃO POR DETERIORAÇÃO DE BIOPRÓTESE, DE ACORDO COM O GRUPO ETÁRIO

\begin{tabular}{|c|c|c|c|}
\hline IDADE & $\begin{array}{l}\text { № DE PACIENTES } \\
\text { QUE NÃO NECES- } \\
\text { SITARAM REOP. }\end{array}$ & $\begin{array}{l}\text { № DE PACIENTES } \\
\text { REOPERADOS }\end{array}$ & TOTAIS \\
\hline$\leqslant 35$ anos & $17(63,0 \%)$ & $10(37,0 \%)$ & 27 (100\%) \\
\hline$>35$ anos & $53(82,0 \%)$ & $12(18,4 \%)$ & $65(100 \%)$ \\
\hline TOTAL & $70(76,1 \%)$ & $22(23,9 \%)$ & 92 \\
\hline & $y=3,62$ & $p=0,057$ & \\
\hline
\end{tabular}

\section{Mortalidade na Substituição da Valva Aórtica}

Os óbitos imediatos ocorreram em 5 pacientes: 2 por insuficiência miocárdica, 1 paciente morreu devido a coagulopatia, 1 em decorrência de pneumotórax hipertensivo e o último teve morte súbita no décimoterceiro dia de pós-operatório. Quatro dos óbitos imediatos ocorreram entre os 47 pacientes que estavam no grupo funcional III e 1 óbito ocorreu entre os 39 pacientes do grupo funcional IV. Houve 1 óbito entre os 22 pacientes portadores de estenose aórtica, 3 óbitos entre os 43 pacientes portadores de insuficiência aórtica e 1 óbito entre os 35 pacientes portadores de dupla lesão aórtica.

A mortalidade tardia durante o período de observação dos pacientes submetidos a substituição da valva aórtica foi de $9,7 \%$ (9/92). Houve perda de seguimento de 3 pacientes e 5 óbitos no pós-operatório imediato.

Os óbitos imediatos e tardios relacionados à bioprótese ocorreram em 7 pacientes, sendo que 3 pacientes tiveram morte súbita ( 1 paciente no pósoperatório imediato e 2 pacientes no tardio), 2 pacientes faleceram por deterioração estrutural, 1 faleceu de dissecção aguda da aorta e outro tinha endocardite infecciosa na bioprótese e faleceu na reoperação (Tabela 3).

\section{TABELA 3}

MORTALIDADE PRÓTESE-RELACIONADA NA SUBSTITUIÇÃO DA VALVA AÓRTICA

$\mathrm{n}=7(7 / 92=7,6 \%)$

\begin{tabular}{ccc}
\hline MORTALIDADE & MORTALIDADE \\
IMEDIATA & TARDIA \\
\hline
\end{tabular}

Mortalidade súbita

Inexplicad

Deterioração Estrutural

da Bioprótese

Dissecção Aguda da Aorta

Endocardite Infecciosa

1

TOTAL

1

\section{Morbidade na Substituição da Valva Aórtica}

Entre os 92 pacientes acompanhados (3 pacientes foram perdidos durante o seguimento e 5 faleceram no pós-operatório imediato), ocorreram degenerações estruturais das biopróteses em 4 pacientes $(10,4 \%)$ entre os 29 que tinham menos de 35 anos na ocasião da primeira operação e 1 paciente $(1,6 \%)$ entre os 63 que tinham mais de 35 anos na ocasião da operação original (Tabela 4).

TABELA 4

VALVA AÓRTICA: DISTRIBUIÇÃO DOS PACIENTES QUE NECESSITARAM OU NÃO REOPERAÇÃO POR DETERIORAÇÃO DA BIOPRÓTESE, DE ACORDO COM O GRUPO ETÁRIO

\begin{tabular}{|c|c|c|c|}
\hline IDADE & $\begin{array}{l}\text { NNo DE PACIENTES } \\
\text { QUE NÃO NECES- } \\
\text { SITARAM REOP. }\end{array}$ & $\begin{array}{l}\text { № DE PACIENTES } \\
\text { REOPERADOS }\end{array}$ & S TOTAIS \\
\hline$\leqslant 35$ anos & $26(89,7 \%)$ & $3(10,4 \%)$ & $29(100 \%)$ \\
\hline$>35$ anos & $62(98,4 \%)$ & $1(1,6 \%)$ & $63(100 \%)$ \\
\hline TOTAL & $88(95,6 \%)$ & $4(4,4 \%)$ & 92 \\
\hline
\end{tabular}

Dois pacientes apresentaram disfunção não estrutural conseqüente de dissecção aguda da aorta do tipo I, de De Beckey. Nesses pacientes, as biopróteses estavam com sua função e as pecto preservados, mas foi necessário substituí-las porque a correção cirúrgica realizada foi feita pela técnica de Bentall e De Bonno, com emprego de tubo valvulado e transferência dos óstios coronarianos.

As conseqüências dos estados mórbidos na substituição da valva aórtica foram analisadas le- 
CARVALHO, M. V. H.; VIEIRA, R. W.; SOUZA, J. M.; OLIVEIRA, S. A. - Desempenho tardio das biopróteses valvulares porcinas. Rev. Bras. Cir. Cardiovasc., 7(1):28-37,1992.

vando-se em conta a necessidade de reoperação e a morbidade relacionadas à presença de bioprótese. Dos 92 pacientes acompanhados, 7 foram reoperados, sendo que 4 por deterioração estrutural da bioprótese e 2 por ocorrência da dissecção aguda da aorta. Um outro paciente sofreu endocardite infecciosa na bioprótese e necessitou ser reoperado (Tabela 4).

Não ocorreram seqüelas permanentes relacionadas à presença de biopróteses.

\section{COMENTÁRIOS}

O objetivo deste trabalho foi analisar o desempenho das biopróteses valvulares porcinas modelo Carpentier-Edwards em posições mitral e aórtica. Foram analisados os primeiros 100 pacientes consecutivos submetidos a substituição da valva mitral isolada e 100 pacientes submetidos a substituição da valva aórtica isolada operados entre maio de 1979 e junho de 1981, permitindo um seguimento pós-operatório médio de 93 meses para os pacientes submetidos a substituição da valva mitral e um seguimento médio de 62 meses para os pacientes submetidos a substituição da valva aórtica. Foram selecionados os pacientes em que a substituição valvar mitral ou aórtica foi um procedimento isolado para evitar que outras doenças cardíacas (por exemplo, coronariopatia) interferissem na evolução desses pacientes.

A mortalidade hospitalar provavelmente não recebeu influência do substituto valvar empregado, uma vez que foi semelhante àquela encontrada quando o substituto valvar é prótese valvular mecânica. Outros grupos de pacientes que utilizaram próteses mecânicas tiveram taxa de mortalidade hospitalar semelhante ${ }^{21,25}$. O fator que mais interferiu na mortalidade imediata foi a falência miocárdica ( $4 \%)$, sendo que este, também, é o principal fator determinante da mortalidade hospitalar quando as próteses mecânicas são usadas ${ }^{7,20,24,25}$.

A análise da morbidade relacionada à bioprótese demonstra que a deterioração estrutural é o evento mórbido mais freqüente e teve sua ocorrência aumentada naqueles pacientes que tinham idade igual ou inferior a 35 anos na ocasião da primeira operação. O fato da deterioração estrutural ser percentualmente mais alta nos pacientes mais jovens já foi constatado por vários autores ${ }^{1-3,8,15,18} \mathrm{e}$, provavelmente, está ligado ao metabolismo do cálcio $6,8,16$. Houve incidência notoriamente maior de degeração estrutural das biopróteses em posição mitral do que das biopróteses em posição aórtica. Entretanto, os pacientes submetidos a substituição da valva aórtica tiveram um período de seguimento menor do que os pacientes com substituição da valva mitral (92 meses para os submetidos a troca mitral e 63 meses para os submetidos a troca aórtica). JAMIESON et alii ${ }^{15}$, em 1988, encontraram maior incidência de deterioração estrutural entre os pacientes submetidos a substituição de valva mitral do que entre os pacientes submetidos a substituição de valva aórtica. CZER et alii ${ }^{10}$, em 1985, usando a prótese de St. Jude, encontraram diferença com significância estatística na mortalidade (maior nos pacientes submetidos a substituição de valva mitral do que nos submetidos a substituição de valva aórtica). Sugere-se que a lesão valvar mitral é mais danosa ao ventrículo esquerdo do que a lesão valvar aórtica.

A incidência de disfunção não estrutural de $1,07 \%$ é o mesmo risco percentual encontrado quando os outros substitutos são empregados, em conformidade com os achados de HAMMOND et alii ${ }^{13}$, em 1987.

A anticoagulação oral não foi utilizada, o que afastou, também, a ocorrência de hemorragias relacionadas ao uso de anticoagulantes e, ainda assim, a incidência de trombo-embolismo foi de 1,07\%. A grande vantagem dos hetero-enxertos porcinos é que a terapia anticoagulante não é necessária em pacientes com ritmo cardíaco sinusal ${ }^{23}$.

A endocardite infecciosa ocorreu em um paciente, que faleceu. A literatura demonstra que a taxa de ocorrência de endocardite é baixa e superponível com os diversos substitutos valvares, mas que a mortalidade é alta, após a sua ocorrência, sendo necessária reoperação precoce como parte essencial da terapêutica ${ }^{2,13}$.

Houve necessidade de reoperação em 22 pacientes, durante o período de seguimento. A deterioração estrutural foi a causa de reoperação em 20 pacientes. Dois outros foram submetidos a reoperação por desprendimento da bioprótese (um paciente) e pela ocorrência de endocardite bacteriana na bioprótese (um paciente). Os três óbitos ocorridos nas reoperaçōes foram de pacientes operados em caráter de emergência, em insuficiência cardíaca grave: um por desprendimento da bioprótese, outro por endocardite infecciosa e o terceiro, no período pós-operatório imediato, por rotura cardíaca após manobras de reanimação. Assim, fica evidente que a deterioração estrutural é um evento marcante, que muda o destino dos pacientes, conforme já assinalado por CHRISTAKIS et alii ${ }^{7}$, em 1985, e SCOTT et alii ${ }^{24}$, em 1985. Uma vez detectados os sinais de deterioração estrutural, a substituição da bioprótese deve ser indicada e realizada precocemente, antes que o paciente caminhe para uma descompensação hemodinâmica que, costumeiramente, é rápida. COHN et alii ${ }^{9}$, em 1986, 
CARVALHO, M. V. H.; VIEIRA, R. W.; SOUZA, J. M.; OLIVEIRA, S. A. - Desempenho tardio das biopróteses valvulares porcinas.

Rev. Bras. Cir. Cardiovasc., 7(1):28-37,1992.

chamam a atenção para o curto espaço de aproximadamente dois meses entre o início da rotura das biopróteses e o aparecimento de sinais de descompensação cardíaca.

Da mesma maneira, no caso da substituição da valva aórtica, a taxa de mortalidade hospitalar não parece ter sido influenciada pelo substituto valvar escolhido. Outros grupos que utilizaram próteses mecânicas tiveram taxa de mortalidade hospitalar semelhante ${ }^{10,19}$. O fator mais marcante da mortalidade hospitalar foi a falência miocárdica, fato este também observado por MITCHELL et alii ${ }^{20}$, em 1987, SETHI et alii ${ }^{25}$, em 1987, GONZALES-LAVIN et alii ${ }^{12}$, em 1989, e LYTLE et alii ${ }^{17}$, em 1989.

A morbidade dos pacientes submetidos ao implante da bioprótese em posição aórtica também foi mais influenciada pela deterioração estrutural, cuja ocorrência foi proporcionalmente mais freqüente naqueles pacientes que tinham idade inferior ou igual a 35 anos na ocasião da cirurgia.
Não houve ocorrência de disfunção não estrutural por refluxo paravalvar, mas dois pacientes tiveram tardiamente dissecção aguda da aorta, após cirurgia para substituição valvar.

A trombose valvar e os acidentes trombo-embólicos não foram observados entre os pacientes com substituição de valva aórtica por bioprótese porcina, apesar de, na nossa série, não ter sido usada a terapêutica anticoagulante. A não utilização dos anticoagulantes deixou os pacientes livres das hemorragias anticoagulante-relacionadas.

As reoperações foram motivadas pela deterioração estrutural em quatro pacientes e por endocardite infecciosa em um paciente. Embora as biopróteses estivessem preservadas em dois pacientes, elas foram substituídas pela necessidade da utilização de condutos valvulados.

A mortalidade prótese-relacionada teve, como fatores determinantes principais, a deterioração estrutural e a morte súbita.

CARVALHO, M. V. H.; VIEIRA, R. W.; SOUZA, J. M.; OLIVEIRA, S. A.- Late performance of porcine valvular bioprostheses. Rev. Bras. Cir. Cardiovasc., 7(1):28-37,1992.

ABSTRACT: The objective of this study was to investigate the late performance of the porcine bioprosthesis Carpentier-Edwards model with emphasis on all morbid and/or lethal events which could be related to its presence. One-hundred consecutive patients who underwent a mitral valve implant and 100 consecutive patients who underwent aortic valve implant were studied. The average tracking period for the former was 93 months and 62 months for the latter. Aproximately $80 \%$ of these patients remained alive during the follow-up time. The survival curves of patients who received mitral valve implant and patients who received aortic valve implant showed an initial decrease due to in-hospital mortality. Then, the curves stabilized showing another decrease about 5 to 6 years later, probably due to structural degeneration. Patients who needed reoperation to substitute the deteriorated bioprosthesis presented a higher mortality rate than those who did not need reoperation. Nevertheless, this difference had not statistic significance. Mortality related to the bioprosthesis was close to $5 \%$ either for patients with bioprosthesis in mitral or in aortic position. Among the 100 patients who received a mitral-position bioprosthesis, 22 needed reoperation to replace it, being the incidence of reoperation higher in those who were under 35 years of age at the time of the first operation. Yet, only 7 among those who received aortic-position bioprosthesis needed reoperation. The thromboembolic complications were rare with use of bioprosthesis although none of the patients received systemic oral anticoagulation. It was concluded that: 1) the use of Carpentier-Edwards porcine bioprosthesis presented satisfactory clinic results concerning in-hospital and late mortality rate, similar not only to other groups but also when other types of valve substitutes are used;2) the structural deterioration is a remarkable event for patients who bear the bioprosthesis, starting basically 6 to 7 years after the implant; 3 ) the structural deterioration of the bioprosthesis modifies patient's fate causing, as a result, reoperation to substitute the implanted bioprosthesis. However, the occurance of such cases does not increase significantly mortality rate .

DESCRIPTORS: heart valves prostheses, biologic. 
CARVALHO, M. V. H.; VIEIRA, R. W.; SOUZA, J. M.; OLIVEIRA, S. A. - Desempenho tardio das biopróteses valvulares porcinas. Rev. Bras. Cir. Cardiovasc., 7(1):28-37,1992.

AGRADECIMENTO: Agradecemos à Prof Cicília Yuko Wada, do IMECC-Unicamp, pela elaboração da análise estatística.

\section{REFERÊNCIAS BIBLIOGRÁFICAS}

1 AMATO, M. C. M.; PORMERANTZEFF, P.; GRIMBERG, M.; BELLOTTI, G.; PILEGGI, F. - Evoluçäo imediata e tardia de pacientes com bioprótese porcina. Arq. Bras. Cardiol., 51:381-384, 1988

2 BLACKSTONE, E. \& KIRKLIN, J. W. - Death and other time related events aftervalve replacement. Circulation, 72:753-767, 1985.

3 BRAILE, D. M. - Prótese valvular de pericárdio bovino: desenvolvimento e aplicação clínica em posição mitral. São Paulo, 1990. [Tese. Doutorado. Escola Paulista de Medicina].

4 BRAIMBRIGE, M.; CHAYEN, J.; BITENSLY, L.; HERASE, D.; JYNGE, P.; DARRACOT, S. - Cold cardioplegia or continuous coronary perfusion? J. Thorac. Surg., 74:900-908, 1974.

5 CARPENTIER, A.; DUBOST, C.; LANE, E. - Continuing improvement in valvular prostheses. J. Thorac. Cardiovasc. Surg., 82:27-42, 1982.

6 CARPENTIER, A.; NASHRAF, A.; CARPENTIER, S.; AHMED, A.; GOUSSEF, N. - Techniques for prevention of calcification of valvular bioprostheses. Circulation, 70 (Supl. 1):165-168, 1984

7 CHRISTAKIS, G. T.; KORMOS, R. L.; WEISEL, R. D.; FREMES, S. E.; TONG, C. P.; HERST J. A.; MICKLEBOROUGH, L. L.; SCULLI, H. E.; GOLDMAN, B. S.; BAIRD, R. J. - Morbidity and mortality in mitral valve surgery. Circulation, 72 (Supl. 2):120$128,1985$.

8 COBANOGLU, A.; JAMIESON, E.; MILLER, D. C.; MCKINLEY, C.; GRUNKEMEYER, G.L.; FLOTEN, S.; MIYAGISHIMA, R. T.; TYERS, G. F.; SHUMWAY, N. E.; STARR, A. - A tri-institucional comparison of tissue and mechanical valve using a patient-oriented definition of "treatment failure". Ann. Thorac. Surg. 43:245$253,1987$.

COHN, L. H.; KOSTER, J. K.; VANDEVANTER, S.; COLLINS, J. J. - The in-hospital risk of replacement of dysfunctional mitral and aortic valves. Circulation, 74 (Supl. 1):153-156, 1986.

10 CZER, L. S. C.; MATLOFF, J; CHAUX, A.; DE ROBERTIS, M.; YOGANATHAN, A.; GRAY, R. J. - A 6 year experience with the St. Jude Medical valve: hemodynamic performance, surgical results, biocompatibility and follow-up. J. Am. Coll. Cardiol., 6:904$912,1985$.

11 EDMUNDS Jr., L. H.; CLARK, R. E.; COHN, L. H. MILLER, C. D.; WEISEL, R. D. - Guidelines for reporting morbility and mortality after cardiac valvular operations. J. Thorac. Cardiovascular. Surg., 96:351-353, 1988.

12 GONZALEZ-LAVIN, L.; GONZALEZ-LAVIN, J.; McGRATH L. B.; AMINI, S.; GRAFT, D. - Factors determining in hospital or later survival after aortic valve replacement. Chest, 95:38-42, 1989.

13

HAMMOND, G. L.; GEHA, A. S.; KOPF, G. S.; HASHIM, S. W. - Biological versus mechanical valves. J. Thorac. Cardiovasc. Surg., 93:182-198, 1987.

JAMIESON, W. R. E.; MUNRO, A. I.; MIYAGISHIMA, R. T.; BURR, L. H.; GEREIN, A. N.; JANUSZ, M. T.; TYERS, F. O.; ALLEN, P. - The Carpentier-Edwards supra anular porcine bioprosthesis. J. Thorac. Cardiovasc. Surg., 96:652-656, 1988.

15 JAMIESON, W. R. E.; ROSADO, L. J.; MUNRO, A. I.; GEREIN, A. N.; BURR, L. H.; MIYAGISHIMA, R. T. JANUSZ, M. T.; TYERS, G. F. O. - Carpentier-Edwards standard porcine bioprosthesis: primary tissue failure (structural valve deterioration) by age groups. Ann. Thorac. Surg., 46:155-162, 1988.

16 LEVI, R. J.; SCHOEN, F. J.; LUND, S. A.; SMITH, M. S. - Prevention of leaflet calcification of bioprosthetic heart valves with diphosphonate injection therapy. $J$. Thorac. Cardiovasc. Surg., 94:551-557, 1987.

LYTLE, B. W.; COSGROVE, D. M.; TAYLOR, P. C.; GOORNASTIC, M.; STEWART, R. W.; GOLDING, L. A. R.; GILL, C. C.; LOOP, F. D. - Primary isolated aortic valve replacement. J. Thorac. Cardiovasc. Surg., 97:675-694, 1989.

18 MAGILLIGAN, D. J.; LEWIS, J. W.; TILLEY, B.; PETERSON E. - The porcine bioprosthetic valve. J. Thorac. Cardiovasc. Surg., 89:499-507, 1985.

19 MILANO, A. D.; BORTOLOTTI, V.; MAZZUCCO, A.; GUERRA, F.; MAGNI, A.; GALLUCCI, V. - Aortic valve replacement with Hancock Standard, Bijörk-Shiley, and Lillehy-Kaster prostheses. J. Thorac. Cardiovasc. Surg., 98:37-47, 1989.

20 MITCHELL, R. S.; MILLER, C.; STINSON, E. B.; OYER, P. E.; JAMIESON, S. W.; BALDWIN, J. C.; SCHUMWAY, N. E. - Significant patient-related determinants of prosthetic valve performance. J. Thorac. Cardiovasc. Surg., 91:807-817, 1986.

21 PERIER, P.; DELOCHE, A.; CHAUVAUD, S.; FABIANI, J. N.; ROSSANT, P.; BESSOU, J. P.; RELLAND, J.; BOUIREZAK, H.; GOMEZ, F.; BLONDEAU, P.; DALLAINES, C.; CARPENTIER, A. - Comparative evaluation of mitral valve repair and replacement with Starr, Bijörk, and porcine valve prostheses. Circulation, 70 (Supl. 1):187-191, 1984. 
CARVALHO, M. V. H.; VIEIRA, R. W.; SOUZA, J. M.; OLIVEIRA, S. A. - Desempenho tardio das biopróteses valvulares porcinas. Rev. Bras. Cir. Cardiovasc., 7(1):28-37,1992.

RAHIMTOOLA, S. - Perspective on valvular heart disease: an update. J. Am. Coll. Cardiol., 14:1-23, 1989.

23

RAHIMTOOLA, S. H. - Valvular heart disease: a perspective. J. Am. Coll. Cardiol., 1:199-215, 1983.

24

SCOTT, W.C.; MILLER, D. C.; HAVERICH, A.; MITCHELL, R. S.; OYER, P. E.; STINSON, E. B.; JAMIESON, S. W.; BALDWIN, J. C.; SHUMWAY, N. E. - Operative risk of mitral valve replacement: discriminant analysis of 1329 procedures. Circulation, 72 (Supl. 2):108$119,1985$.

25 SETHI, G. K.; MILLER, D. C.; SOUCHECK, J.; OPRIAN, C.; HENDERSON, W. G.; HASSAN, Z.; FOLLAND, E.; KRURI, S.; SCOTT, S. M.; CURCHFIELD, C.; HARMMENMEISTER, K. E. - Clinical hemodinamyc, and angiographic predictor of operative mortality in patients undergoing single valve replacement. $J$. Thorac. Cardiovasc. Surg., 93:884-897, 1987. 